7. Gandacu D, Glazer Y, Anis E, Karakis I, Warshavsky B, Slater P, et al. Resurgence of cutaneous leishmaniasis in Israel, 2001-2012. Emerg Infect Dis. 2014;20:1605-11. http://dx.doi.org/10.3201/ eid2010.140182

8. Solomon M, Greenberger S, Baum S, Pavlotsky F, Barzilai A, Schwartz E. Unusual forms of cutaneous leishmaniasis due to Leishmania major. J Eur Acad Dermatol Venereol. 2016;30:11715. http://dx.doi.org/10.1111/jdv.13220

9. Ehehalt U, Schunk M, Jensenius M, van Genderen PJ, Gkrania-Klotsas E, Chappuis F, et al. Leishmaniasis acquired by travellers to endemic regions in Europe: a EuroTravNet multi-centre study. Travel Med Infect Dis. 2014;12:167-72. http://dx.doi.org/10.1016/j.tmaid.2013.12.003

Address for correspondence: Perry J.J. van Genderen, Institute for Tropical Diseases, Harbour Hospital, Haringvliet 72, 3011 TG Rotterdam, the Netherlands; email: p.van.genderen@havenziekenhuis.nl

\section{Molecular Evidence of Oysters as Vehicle of Norovirus GII.P17-GII.17}

\section{Lasse Dam Rasmussen, Anna Charlotte Schultz, Katrine Uhrbrand, Tenna Jensen, Thea Kølsen Fischer}

Author affiliations: Statens Serum Institute, Copenhagen,

Denmark (L.D. Rasmussen, T.K. Fischer); Technical University of Denmark, Kongens Lyngby, Denmark (A.C. Schultz, K. Uhrbrand); Danish Veterinary and Food Administration, Glostrup, Denmark (T. Jensen); University of Southern Denmark, Odense, Denmark (T.K. Fischer)

DOI: http://dx.doi.org/10.3201/eid2211.161171

To the Editor: Norovirus is the world's leading cause of nonbacterial acute gastroenteritis (1). Since their emergence, GII.P17-GII.17 noroviruses have replaced the GII.4 Sydney 2012 variant as the dominating norovirus genotype in parts of Asia (2), although they have been detected only sporadically, in a limited number, on other continents (3).

The major reservoir(s) of GII.17 that contribute to transmission are unknown, but it has been suggested that oysters and other bivalve shellfish are common vehicles for transmission of the emerging GII.17 viruses (2). In this study, we demonstrate the link between oysters and human disease by presenting molecular evidence of norovirus GII. P17-GII.17 in Denmark causing acute gastroenteritis, characterized by the sudden onset of vomiting with or without diarrhea after consumption of oysters. We further document molecular evidence providing linkage between norovirus detected in fecal samples from patients and food samples from imported oysters.
During January 23-February 4, 2016, acute gastroenteritis developed in 58 of 67 persons who consumed oysters served on 18 separate occasions at 8 different restaurants and a private party, with onset of symptoms within 24-40 hours after the patients ate oysters. All oysters originated from 2 distinct oyster lots provided by 1 wholesaler in France and distributed by 1 wholesaler in Denmark. Oysters from both lots were harvested off the coast of La Rochelle, France.

In Denmark, submitting fecal samples in connection with foodborne outbreaks is voluntary. A total of 5 samples from 3 cases representing 2 different parties were submitted to the National Virus Surveillance Laboratory at Statens Serum Institut (Copenhagen, Denmark) for norovirus analysis. In addition, 4 samples of oysters from the same producer in France were sent to the National Food Institute, Technical University of Denmark (Kongens Lyngby, Denmark), for norovirus analysis. Two samples, an opened (sample A) box and an unopened (sample B) box collected at one of the restaurants involved in the outbreak, contained oysters from 1 batch (I), and another 2 samples (C and D) contained oysters from a separate batch (II) collected at the wholesale level.

Norovirus in fecal samples was detected and polymerase (open reading frame [ORF]1) and capsid (ORF2) gene regions were amplified as described elsewhere (4). Sequencing was performed by using an ABI 3500 genetic analyzer (Thermo Fisher Scientific, Nærum, Denmark). A fragment of 1,111 nt spanning the ORF1-ORF2 junction was amplified and sequenced by using forward ORF1 primer JV12 and reverse ORF2 primer G2SKR (4). Genotyping was performed by using the Web-based norovirus typing tool NoroNet (http://www.rivm.nl/mpf/norovirus/typingtool) (5).

We found that all fecal samples contained norovirus belonging to genogroup II (GII). Sequencing of the polymerase region was successful in 4 of the 5 samples; all 5 samples were sequenced in the capsid region. Typing of the sequences indicated GII.P17 and GII.17, respectively. Furthermore, PCR and sequencing of the long fragment covering the ORF1-ORF2 junction were successful in 2 samples, both genotyping as GII.P17-GII.17. Sequences had high homology (99.91\%) to several Asian strains, such as Hu/GII.P17_GII.17/KR/2014/CAU-265 (6).

Oysters were analyzed for norovirus (7), and the capsid region of the detected strains was sequenced (8). Samples $\mathrm{A}, \mathrm{B}$, and $\mathrm{C}$ contained norovirus GI, whereas all 4 samples (A-D) contained norovirus GII. GI.2 was identified in 1 sample (B), and GII.17 was identified in all samples (A-D). The GII.17 sequences obtained from samples A-C showed $100 \%$ homology, whereas the sequence identified in sample $\mathrm{D}$ varied at 3 positions.

Comparisons of the capsid sequences obtained from 4 human samples and oyster samples A-C showed 100\% 
homology. The last of the human sequences differed at 2 positions owing to mixed bases. The chromatogram showed equal intensities at both positions $\mathrm{C}$ and $\mathrm{T}$ (Figure, http://wwwnc.cdc.gov/EID/article/22/11/16-1171-F1. $\mathrm{htm})$. This finding indicates that quasispecies might be present. These positions in the human sequence coincided perfectly with 2 of the 3 positions found to vary in the sequence obtained from oyster sample D. All 3 base variations were a replacement of a $\mathrm{C}$ with a $\mathrm{T}$ (Figure), which further supports the presence of quasispecies. However, in this setup, it was not possible to prove the origin of these closely related species. Whole-genome sequencing using next-generation sequencing would be a way to prove the simultaneous presence of all quasispecies in relevant samples.

Since the emerging of GII.P17-GII.17 in Asia, sporadic cases have been reported worldwide $(3,9)$. In this study, we established a direct molecular link between a common food source and a series of acute gastroenteritis outbreaks. Even though these represent European outbreaks, our results show that oysters act as vehicles for the rapid spread of emerging noroviruses to distant geographic areas. Furthermore, we document that quasispecies of GII.P17-GII.17 might occur simultaneously in a host. This finding should be considered in future molecularepidemiologic outbreak investigations.

\section{Acknowledgments}

We thank Joanna Zeitman Amenuvor and Resadije Idrizi for excellent technical assistance.

The study of the oysters was partially funded by Aquavalens (EU FP7-KBBE-2012-6) (http://aquavalens.org/).

Dr. Rasmussen is a molecular biologist working as a senior scientist at Statens Serum Institut. His primary research interest is emerging viruses.

\section{References}

1. Atmar RL, Estes MK. The epidemiologic and clinical importance of norovirus infection. Gastroenterol Clin North Am. 2006;35:27590, viii. http://dx.doi.org/10.1016/j.gtc.2006.03.001

2. Lu J, Fang L, Zheng H, Lao J, Yang F, Sun L, et al. The evolution and transmission of epidemic GII.17 noroviruses. J Infect Dis. 2016;214:556-64. http://dx.doi.org/10.1093/infdis/jiw208

3. de Graaf M, van Beek J, Vennema H, Podkolzin AT, Hewitt J, Bucardo F, et al. Emergence of a novel GII.17 norovirusend of the GII.4 era? Euro Surveill. 2015;20:21178. http://dx.doi.org/10.2807/1560-7917.ES2015.20.26.21178

4. Franck KT, Fonager J, Ersbøll AK, Böttiger B. Norovirus epidemiology in community and health care settings and association with patient age, Denmark. Emerg Infect Dis. 2014; 20:1123-31. http://dx.doi.org/10.3201/eid2007.130781

5. Kroneman A, Vennema H, Deforche K, van der Avoort H, Peñaranda S, Oberste MS, et al. An automated genotyping tool for enteroviruses and noroviruses. J Clin Virol. 2011;51:121-5. http://dx.doi.org/10.1016/j.jcv.2011.03.006

6. Dang Thanh H, Than VT, Nguyen TH, Lim I, Kim W. Emergence of norovirus GII.17 variants among children with acute gastroenteritis in South Korea. PLoS One. 2016;11:e0154284. http://dx.doi.org/10.1371/journal.pone.0154284

7. Franck KT, Lisby M, Fonager J, Schultz AC, Böttiger B, Villif A, et al. Sources of calicivirus contamination in foodborne outbreaks in Denmark, 2005-2011: the role of the asymptomatic food handler. J Infect Dis. 2015;211:563-70. http://dx.doi.org/10.1093/ infdis/jiu479

8. Müller L, Schultz AC, Fonager J, Jensen T, Lisby M, Hindsdal K, et al. Separate norovirus outbreaks linked to one source of imported frozen raspberries by molecular analysis, Denmark, 2010-2011. Epidemiol Infect. 2015;143:2299-307. http://dx.doi.org/10.1017/ S0950268814003409

9. de Graaf M, van Beek J, Koopmans MPG. Human norovirus transmission and evolution in a changing world. Nat Rev Microbiol. 2016;14:421-33. http://dx.doi.org/10.1038/nrmicro.2016.48

Address for correspondence: Lasse Dam Rasmussen, Afdeling for Mikrobiologisk Diagnostik og Virologi, Sektor for Diagnostik og Infektionskontrol, Artillerivej 5, DK-2300 Copenhagen S, Denmark; email: lara@ssi.dk

\section{Recent Chikungunya Virus Infection in 2 Travelers Returning from Mogadishu, Somalia, to Italy, 2016}

\section{Lorenzo Zammarchi, Claudia Fortuna, Giulietta Venturi, Francesca Rinaldi, Teresa Capobianco, Maria Elena Remoli, Gian Maria Rossolini, Giovanni Rezza, Alessandro Bartoloni}

Author affiliations: Università Degli Studi di Firenze, Florence, Italy (L. Zammarchi, F. Rinaldi, G.M. Rossolini, A. Bartoloni); Azienda Ospedaliero-Universitaria Careggi, Florence (L. Zammarchi, T. Capobianco, G.M. Rossolini, A. Bartoloni); Istituto Superiore di Sanità, Rome, Italy (C. Fortuna, G. Venturi, M.E. Remoli, G. Rezza); Università di Siena, Siena, Italy (G.M. Rossolini); Fondazione IRCCS Don Carlo Gnocchi, Florence (G.M. Rossolini)

\section{DOI: http://dx.doi.org/10.3201/eid2211.161225}

To the Editor: Since chikungunya virus (CHIKV) was first isolated in 1952 (in Tanzania), outbreaks have occurred every 7-20 years in countries in Africa and Asia, and since 2013, it has been identified in the Americas $(1,2)$. However, no cases have been reported from the Horn of Africa $(3,4)$. We confirmed CHIKV infection acquired in 2016 by 2 travelers to Somalia who returned to Italy.

In June 2016, a Somali woman (patient 1) was referred to the Infectious and Tropical Diseases Unit, Careggi University Hospital, in Florence, Italy, because of severe diffuse 